1. First Author:

First Name: Jingde

Last Name: Li

Academic Degree: PhD Candidate

Affiliation: School of Civil and Resource Engineering, the University of Western Australia

Address: 35 Stirling Highway, Crawley WA 6009, Australia

E-mail Address: jingde.li@ research.uwa.edu.au

2. Corresponding Author:

First Name: Guowei

Last Name: Ma

Academic Degree: PhD (Professor)

Affiliation: School of Civil and Resource Engineering, the University of Western Australia

Address: 35 Stirling Highway, Crawley WA 6009, Australia

E-mail Address: ma@civil.uwa.edu.au

3. Other Author:

First Name: Madhat

Last Name: Abdel-jawad

Academic Degree: PhD (Dirctor)

Affiliation: GexCon AS Australia

Address: 8/64 Fitzgerald Street, Northbridge WA 6003, Australia

E-mail Address: madhat@gexcon.com

4. Other Author:

First Name: Hong

Last Name: Hao

Academic Degree: $\mathrm{PhD}$ (Professor)

Affiliation: School of Civil \& Mechanical Engineering, Curtin University

Address: Kent Street, Bentley, WA 6120, Australia

E-mail Address: hong.hao@ curtin.edu.au 


\title{
Evaluation of gas explosion overpressures at configurations with
} irregular-arranged obstacles

Jingde $\mathrm{Li}^{1, \text { a }}$, Guowei Ma ${ }^{1, \mathrm{~b}}$, Madhat Abdel-jawad ${ }^{2, \mathrm{c}}, \mathrm{Hong} \mathrm{Hao}^{3, \mathrm{~d}}$

\author{
${ }^{1}$ School of Civil and Resource Engineering, The University of Western Australia,
} 35 Stirling Highway, Crawley WA 6009, Australia

${ }^{2}$ GexCon Australia, 8/64 Fitzgerald Street, Northbridge WA 6003, Australia ${ }^{3}$ School of Civil \& Mechanical Engineering, Curtin University, Kent Street, Bentley, WA 6120, Australia ajingde.li@ research.uwa.edu.au, ${ }^{\mathrm{b}} \mathrm{ma} @$ civil.uwa.edu.au, ${ }^{\mathrm{c}}$ madhat@gexcon.com, dhong.hao@curtin.edu.au

\section{Abstract}

Rapid analytical methods for the calculation of gas explosion overpressures in confined and congested regions are of great value where a benchmark value is sought rather than a time consuming detailed analysis obtainable by Computational Fluid Dynamics (CFD). While earlier correlations have been compared directly to experiments, the geometries used were often simplistic and displayed homogeneity in confinement and congestion. Realistic geometries typically display a high degree of inhomogeneity in confinement and congestion. Here we examine geometries where the confinement and congestion were deliberately varied such that some of the geometries possessed inhomogeneity of both parameters. Little experimental data exists for such configurations and hence we examine these configurations using CFD. The CFD overpressure predictions at various target locations for 400 scenarios are compared with the results from a newly derived correlation and the correlation of the Guidance for the 
Application of the Multi-Energy method (GAME). It is found that the overpressure predictions obtained using the correlation still better agrees with the CFD modelling results compared with the GAME correlation suggesting. To show the importance of increased accuracy in these cases, a structural damage level evaluation process is used to place the damage levels for 4 monitor points on a p-i curve and the results show that often these damage levels are near critical, demonstrating the need for improved accuracy.

Keywords: obstacles, VCEs, gas explosion, irregularity, overpressure, turbulence

\section{Introduction}

Numerous vapour cloud explosions (VCE), and dust explosions occur each year worldwide. The vapour cloud explosion, is defined as "an explosion resulting from an ignition of a premixed cloud of flammable vapour, gas or spray with air, in which flames accelerate to sufficiently high velocities to produce significant overpressure" (Mercx \& van den Berg, 2005). These represent one of the most significant hazards in the chemical process industry. Due to the large overpressures generated from the VCEs, it can result in potential environmental damage and enormous financial loss in addition to injury and loss of life. As a result, it is of great importance to assess risk at major hazard facilities accurately.

Deflagration is a combustion wave propagating at subsonic velocities relative to the unburned gas immediately ahead of the flame. Detonation is defined as a supersonic combustion wave (i.e. the detonation front propagates into unburned gas at a velocity higher than the speed of sound in front of the wave) (Bjerketvedt et al., 1997). In this paper we consider the MERGE and EMERGE projects (EMEG, 1997; Harris \& 
81 Wickens, 1989; Mercx et al., 1995; Schumann et al., 1993; Wingerden, 1988, 1989), which were conducted to investigate the mechanism of the gas explosions. Using experiments to evaluate risk for each industrial facility is impractically expensive due to the numerous variations of geometry detail, size and inventory composition and size in industrial explosion scenarios. Cost constraints mean that experiments performed so far have been scaled down in size and simplifications were applied. The scaling factor may result in inherent uncertainties for experimental results and it is sometimes difficult to even quantify the impact of the simplifications used in these experiments.

Based on experiments, some theoretical methods were developed, such as the widely used approach TNO Multi-Energy Method (MEM)(Vandenberg, 1985). MEM is a simple phenomenological approach to estimate overpressures from approximated vapour cloud explosion scenarios. However, MEM has some clear limitations. Firstly, MEM was derived based on limited scale experiments which results in uncertainties in the prediction of pressures for large-scale explosion scenarios. Secondly, the directional effects for explosions due to localised confinement and congestion are not accounted for, as the results output by MEM are radial in nature. Finally and importantly, the nearfield gas explosion overpressure cannot be predicted via the multi-energy approach with any reasonable accuracy and it relies on an input estimate of the strength of the explosion which can be either significantly underestimated or significantly overestimated: both leading to unsatisfactory results.

An improvement on MEM is the GAME approach (Eggen, 1995). Specifically, several parameters regarding the directional effects and gas properties, such as the degree of geometry size, congestion, gas mixture and the laminar flame speed, among others, are 
investigated in the GAME approach, however, the derivation of the GAME correlations are based on the phenomenological analysis of the experimental programs which were arranged with regular obstacles. When it comes to cases with inhomogeneous congestion and confinement, the accuracy of the GAME correlations has not been adequately tested against a standard.

Consequently, at the present time, many of the vapour cloud explosion analyses are increasingly being carried out using the Computational Fluid Dynamics (CFD) tools (Marangon et al., 2007). Because it agrees with experiments to a greater degree than analytical studies, the CFD approach is considered a robust numerical tool based on finite volume solutions and the 'physical' models of combustion process to predict gas explosion overpressure. In particular, some CFD solvers can capture the flame acceleration and venting of the overpressure build-up for gas clouds in irregularly patterned obstacles which have significant effects on overpressures.

However CFD is time consuming and expensive and in addition requires a degree of expertise in its application for meaningful results and there is still significant need for rapid approximate methods for benchmarking such events that can be later targeted, if necessary with detailed CFD analysis. In this paper we used the detailed CFD methodology as a benchmark to further investigate a previously suggested rapid solution - a confinement specific correlation (CSC) (Li et al., 2014).

Here the highly validated commercial CFD software FLACS (GexCon, 2011) was utilized in the evaluation, of a benchmarking correlation previously proposed (Li et al., 2014). The software was used to test the robustness of the correlation particularly its 
ability to predict overpressures for cases with variation of a few fundamental parameters including confinement and congestion driven flame propagation, a range of practical modules with irregularly arranged obstacles and confinement ratios were assessed by means of the previous proposed correlation (Li et al., 2014). After the evaluation of the overpressure, the data of the pressure-impulse ( $p-i)$ was also analysed in this study which is able to be used for structural damage prediction.

\section{Simulation Methodology}

\section{The FLACS Software}

In order to extend the range of conditions for the correlation of (Li et al., 2014), the results for overpressure are compared with the results using the commercial software FLACS (GexCon, 2011) for conditions not previously considered. FLACS (GexCon, 2011) is a finite volume solver that solves the Reynolds averaged mass, momentum and energy balance equations, with special schemes for supersonic flows and a database of chemical kinetics. The mathematical model of FLACS (GexCon, 2011) is given in (Arntzen, 1998; Ferrara et al., 2006; Hjertager, 1984, 1993).

For a general variable, the differential equation, which is based on Reynolds averaged mass, momentum and energy balance equations, may be expressed as follows using standard symbols:

$$
\frac{\partial}{\partial t}(\rho \varphi)+\frac{\partial}{\partial x_{j}}\left(\rho u_{j} \varphi\right)-\frac{\partial}{\partial x_{j}}\left(\Gamma_{\varphi} \frac{\partial \varphi}{\partial x_{j}}\right)=S_{\varphi} ; \Gamma_{\varphi}=\frac{\mu_{e f f}}{\sigma_{\varphi}}
$$


where $f$ denotes a general variable, $\rho$ is the gas mixture density, $x_{j}$ is the coordinate in $j$-direction, $u_{j}$ is the velocity component in $j$-direction, $\Gamma_{\varphi}$ is the effective (turbulent) diffusion coefficient, $\mu_{e f f}$ is the effective turbulence viscosity and $S_{\varphi}$ is a source term.

A summary of all the governing equations needed for a typical reactive gas dynamic calculation are presented below.

The state equation of an ideal gas:

$$
p W=\rho R T
$$

where $p$ is the pressure, $R$ is the universal gas coefficient $T$ is temperature and $W$ is the molar weight of the gas mixture.

The continuity equation:

$$
\frac{\partial \rho}{\partial t}+\frac{\partial}{\partial x_{j}}\left(\rho u_{j}\right)=0
$$

The momentum balance equation:

$$
\frac{\partial}{\partial t}\left(\rho u_{i}\right)+\frac{\partial}{\partial x_{j}}\left(\rho u_{j} u_{i}\right)=-\frac{\partial p}{\partial x_{i}}+\frac{\partial}{\partial x_{j}}\left(\sigma_{i j}\right)
$$

The energy balance equation:

$$
\frac{\partial}{\partial t}(\rho h)+\frac{\partial}{\partial x_{j}}\left(\rho u_{j} h\right)=\frac{\partial}{\partial x_{j}}\left(\Gamma_{h} \frac{\partial h}{\partial x_{j}}\right)+\frac{\partial p}{\partial t}+u_{j} \frac{\partial p}{\partial x_{j}}
$$

where $\sigma_{i j}$ is the flux of momentum and $h$ is the enthalpy.

The solver accounts for dissipation of turbulent kinetic energy with a modified k- $\varepsilon$ model (Arntzen, 1998; Hjertager, 1993).

The equation for turbulent kinetic energy: 


$$
\frac{\partial}{\partial t}(\rho k)+\frac{\partial}{\partial x_{j}}\left(\rho u_{j} k\right)=\frac{\partial}{\partial x_{j}}\left(\frac{\mu_{e f f}}{\sigma_{k}} \frac{\partial k}{\partial x_{j}}\right)+G-\rho \varepsilon ; G=\sigma_{i j} \frac{\partial u_{j}}{\partial x_{i}}
$$

177 The equation for dissipation of turbulent kinetic energy:

$$
\frac{\partial}{\partial t}(\rho \varepsilon)+\frac{\partial}{\partial x_{j}}\left(\rho u_{j} \varepsilon\right)=\frac{\partial}{\partial x_{j}}\left(\frac{\mu_{e f f}}{\sigma_{\varepsilon}} \frac{\partial \varepsilon}{\partial x_{j}}\right)+1.44 \frac{\varepsilon}{k} G-1.79 \rho \frac{\varepsilon^{2}}{k}
$$

179 where $G$ is the generation rate of turbulence.

180

181

182

183 correction algorithm (Patankar, 1980). in the $\mathrm{x}_{\mathrm{j}}$-direction.

The combustion process is treated as a single step irreversible reaction with finite reaction rate between fuel and oxidant. The reaction scheme results in mixture composition being determined by solving for only two variables, namely mass fraction of fuel $m_{f u}$, and the mixture fraction $f$ (Hjertager, 1984):

$$
\begin{aligned}
\frac{\partial}{\partial t}\left(\rho m_{f u}\right)+\frac{\partial}{\partial x_{j}}\left(\rho u_{j} m_{f u}\right) & =\frac{\partial}{\partial x_{j}}\left(J_{f u, j} \frac{\partial \varepsilon}{\partial x_{j}}\right)+R_{f u} \\
\frac{\partial}{\partial t}(\rho f)+\frac{\partial}{\partial x_{j}}\left(\rho u_{j} f\right) & =-\frac{\partial}{\partial x_{j}}\left(J_{f, j}\right)
\end{aligned}
$$

where $R_{f u}$ is the time mean rate of combustion of fuel, $J_{f u, j}$ and $J_{f, j}$ are the diffusive fluxed

FLACS (GexCon, 2011) solves the equations above such that the overpressures from previous time step, the momentum equation gives a velocity field, which will be corrected along with the updated pressure and density field by implementing a pressure

$$
\text { (Patankar, 1980). }
$$


The factors of the fuel density, the flame radius, the initial laminar flame speed of fuel play important roles in the combustion of an explosion, thereby resulting in the development of the overpressure.

Overall, influence of all parameters on the formation of explosion pressures including the mechanism of turbulent reactive gas dynamics, combustion processes and the geometry of the configurations are taken into account in the methodology of the CFDbased solver - FLACS (GexCon, 2011).

\section{Geometry model}

The cases examined in this paper are analysed using CSC and also modelled using FLACS (GexCon, 2011). These are cases of large-scale geometries at scales encountered in industrial scenarios in process safety. Examples are artificial and realistic models in Fig. 1 with sizes of $90 \times 45 \times 15(\mathrm{~m})$ and $80 \times 50 \times 50(\mathrm{~m})$, respectively. The artificial geometries in this study were modelled with mixed obstacle arrangement patterns, obstacle diameters and confinement ratios and one realistic module truncated from a LNG (Liquefied Natural Gas) train (Fig. 1 (b) ) was also investigated.

Both propane and methane VCEs were modelled in this paper. The ambient temperature and pressure were set as $26^{\circ} \mathrm{C}$ and $101 \mathrm{kPa}$, respectively. Eulerian boundary conditions of the domain were used and the $\mathrm{BC}$ pressure was set to be equal to the ambient pressure.

Walls and decks were assumed to be unyielding during the entire explosion, i.e. rigid walls remain in place even for the largest explosion loads. FLACS (GexCon, 2011) is based on several subgrid models which require careful observation of some best 
practice guidelines. These include the use of cubical grid cells in the combustion region were applied in order to diminish the deviations of flame propagation and pressures; the aspect ratio of the grid is controlled to within $20 \%$ and grid cells smaller than $5 \mathrm{~cm}$ were avoided to ensure the accurate results.

For purpose of extracting the pressures, monitor points were defined at specific locations in the simulation domain where variables including volume blockage ratio (VBR), the distance of flame propagation, the characteristic average obstacle diameter are to be monitored. For instance, as shown in Fig. 1 (a), the gas cloud was ignited at the edge centre of the configuration; the monitor points were then placed along the direction of flame propagation to obtain the pressures at the increasing of the flame propagation distance. And for each simulation in this paper, more than 30 monitor points were assigned according to the grid arrangement.

\section{Evaluation of the irregular-arranged configurations subjected to gas explosion}

The confinement specific correlation (CSC) derived in previous work (Li et al., 2014) is used to independently predict the overpressures for similar cases with irregular arrangement of obstacles. The dimensionless and confinement specific correlation regarding the parameters of confinement, volume blockage ratio, the average obstacle, laminar flame velocity and gas density is given by:

$$
\frac{\Delta \mathrm{P}_{\mathrm{o}}}{P_{\text {air }}}=0.037 \cdot e^{8.5 C_{m}} \cdot\left[1.6 \ln \left(V B R_{t}\right)+6\right] \cdot\left(\frac{L_{f d}}{H}\right)^{2.2} \cdot\left(\frac{D}{H}\right)^{-1.5} \cdot\left(\frac{\rho_{\text {gas }}}{\rho_{\text {air }}}\right)^{0.5} \cdot\left(\frac{S_{l}}{S_{s}}\right)^{2}(10)
$$

where:

$\Delta \mathrm{P}_{\mathrm{o}}=$ the escalation overpressure $[$ barg $]$,

$$
\mathrm{P}_{\mathrm{air}}=1 \text { standard atmospheric pressure } 101.325 \mathrm{kPa}[1 \mathrm{barg}] \text {, }
$$


$\mathrm{D}=$ the average obstacle diameter $[\mathrm{m}]$,

$\mathrm{L}_{\mathrm{fd}}=$ the direct distance from the ignition location to the target point $[\mathrm{m}]$,

$\mathrm{S}_{1}=$ the laminar flame speed of the flammable gas $[\mathrm{m} / \mathrm{s}]$,

$S_{S}=$ the speed of sound $[\mathrm{m} / \mathrm{s}]$,

$\mathrm{C}_{\mathrm{m}}=$ the confinement ratio,

$\mathrm{VBR}_{\mathrm{t}}=$ the volume blockage ratio of configuration region from the ignition point

to the target,

$\rho_{\text {gas }}=$ mass density of gas $\left(\mathrm{kg} / \mathrm{m}^{3}\right)$ (the gas density is assumed ideally under one standard atmosphere pressure at normal temperature 26 degrees in this study), $\rho_{\text {air }}=$ mass density of air $\left(\mathrm{kg} / \mathrm{m}^{3}\right)$,

$H=$ the height of the configuration (m).

\section{Definition of regularity and irregularity of Confinement and Congestion}

In this study, we examined regular and irregular arrangements of congestion and confinement. This subsection describes both types of geometries.

In terms of the congestion, the artificial module in Fig. 1 (a) features uniform obstacle diameter and a regular pattern of obstacles. By contrast, the module 1 and module 4 in Fig. 3 were modelled with irregularities. And more importantly, unlike the previous study ( $\mathrm{Li}$ et al., 2014) where the simulations are modules extracted from an existing LNG (Liquefied Natural Gas) train; they are composed of realistic layouts of structural components with random irregularities. The geometry displayed in Fig. 3 of this paper are artificial modules with controllable irregularities, for example, from module 1 to 4 , they are intentionally organized with increasing obstacle diameters, equidistant 
separation distances and mixed intersecting obstacle arrangements, etc. Additionally, those artificially arranged irregular modules in this paper are large-scale modules whilst those artificial ones in the previous study (Li et al., 2014) are in small-scale.

Using the definition of confinement in the previously proposed paper (Li et al., 2014), all simulations were conducted under the configurations with the parallel plates in semi3D overpressure expansion; the confinement ratio is characterized as the ratio of the blocked area on the top and bottom plates over the total area of the top and bottom surfaces. Therefore, a configuration covered with two solid top and bottom plates, such as the module in Fig. 2 (a), is considered to be fully confined in the z- direction; and the one without top plate is defined as open in the $+\mathrm{z}$-direction, as seen Fig. 2 (c). In this study, the partial confinement between the open air and the full confinement is used to test the correlations under conditions of irregular confinement.

\section{Application of the CSC to the irregular-arranged modules}

By using the CSC, overpressures were estimated for configurations with congestion of an irregular arrangement subjected to vapour cloud explosions and the results are described in this section. As seen in Fig. 3, four modules with inhomogeneous obstacles plus one realistic module were modelled here to simulate 400 new explosions for this study. Four of the modules are of highly confined configurations. In the explosion models, a stoichiometric flammable gas cloud was used to fill the obstacle configurations; methane and propane are both used as fuels in this study. The parameters are shown in Table 1. 
Fig. 4 shows the correlation pressure predictions on the $\mathrm{x}$ - axis against the pressures calculated with FLACS (GexCon, 2011) on the $y$ - axis. The R-squared $\left(\mathrm{R}^{2}\right)$ value is extracted for each of these cases. As seen in Fig. 4, the R-squared value for each simulation model is between 0.66 and 0.90 , which shows the CSC correlation applies to practical geometries of greatly varying confinement ratios as well as irregular pattern of VBR and varying obstacle diameters in the configurations. The results from the CSC correlation were also compared to results from the Guidance for the Application of the Multi-Energy method (GAME) correlation (Eggen, 1995).

The GAME correlation below was used to determine the gas explosion overpressure for the modules in Fig. 3 with confinement between parallel plates.

$$
\Delta \mathrm{P}_{\mathrm{o}}=3.38 \cdot\left(\frac{\mathrm{VBR} \cdot \mathrm{L}_{\mathrm{f}}}{\mathrm{D}}\right)^{2.25} \mathrm{~S}_{\mathrm{l}}^{2.7} \cdot \mathrm{D}^{0.7}
$$

where VBR is the volume blockage ratio defined as the ratio of the total volume of the obstacles inside an obstructed region, $L_{f}$ is the maximum distance of flame propagation obtained by assuming $L_{f}$ equal to the radius of a hemisphere with a volume equal to the volume of the configuration, $D$ is the averaged obstacle diameter based on the entire configuration, $S_{l}$ is the laminar flame speed of the flammable gas

The GAME correlation is seen to be generally, but not always conservative in the determination of the overpressure for cases with artificially homogenous congestion. When applied to geometries (Fig. 3) with irregularities of confinement and congestion, the overall comparison results, seen in Fig. 5, give a poor agreement with the FLACS results, specifically, the data obtained by means of the GAME correlation tend to overestimate the overpressure significantly whereas the CSC correlation result agrees well with FLACS simulations, Fig. 5. 
317 The GAME correlation was derived from MERGE experiments (EMEG, 1997; Harris

318 \& Wickens, 1989; Mercx et al., 1995; Schumann et al., 1993; Wingerden, 1988, 1989)

319 which possesses the idealized obstacles with average diameter and homogeneously 320 distributed in the configuration, the volume blockage ratio and confinement ratio are

321 regularly patterned. In this study, we examine the performance of the GAME 322 correlation for cases where the irregularities of the obstacles as well as high degrees of 323 confinement are characteristics of geometry. This has not been adequately tested using 324 GAME correlation up till this point. The CSC correlation is derived based on the CFD 325 coded software - FLACS (GexCon, 2011), the parameters regarding the geometrical 326 detail and the turbulent reactive gas dynamics mechanism are accounted for, hence this 327 approach better models the inhomogeneous configurations where the turbulence 328 generation/degeneration and the burning velocity acceleration/ deceleration are key 329 factors in the variation of the congestion and confinement.

\section{Rapid prediction of structural damage}

332 The CSC correlation has undergone validation ( $\mathrm{Li}$ et al., 2014) with very good 333 agreement with pressures predicted using CFD modelling. In this study we also add a 334 rapid structural damage level prediction process; two different simulation 335 configurations with 8 well-located monitor points were numerically modelled using using FLACS (GexCon, 2011) as the case studies shown below, the pressure vs. time history data was obtained for the specific structure members at those monitor points.

339 As seen in Fig. 7, the overpressure figures are observed from the fully congested 340 configuration Fig. 6 (a) and the configuration with a sufficient separation distance Fig. 
6 (b). For both configurations, the explosion occurs from the centre of the left module as illustrated in Fig. 1 (a), the flame propagates through the fuel away from the ignition point till the fuel exhausted, the monitor points 1 to 4 are place in the centre along the flame propagation direction from left to right. It is noted in Fig. 7 (a) that the magnitude of the maximum overpressure increases from $125 \mathrm{kPa}$ to $230 \mathrm{kPa}$ as the flame path from the ignition through congestion increases, the maximum overpressure is seen at monitor point 4 .

The phenomenon observed above is attributed to flame acceleration which is described in (Bjerketvedt et al., 1997; Eggen, 1995; Li et al., 2014), the geometry of the gas explosion scenario and flame propagation distance both contribute the development of the flame acceleration and overpressure. In a gas explosion scenario, turbulence is generated when the flame interacts with the obstacles, which results in the flame acceleration and the generation of more turbulence as the flame propagates further in the congested area: a self-feeding mechanism increasing flame speed and thereby increasing the overpressure. This is in contrast to an explosion pressure field from a scenario using explosives where the maximum blast load is seen at the minimum standoff distance decreasing with distance from ignition point.

However, if a flame propagates in a premixed air-fuel cloud in an uncongested open space, as seen in Fig. 7 (b), the phenomenon of flame acceleration does not continue in the open uncongested space. The separation space in Fig. 6 (b) reduces the congestion and intensity of turbulence which results in the decrease of the overpressure. An explosion generated with explosives is not affected by a separation space in the same 
manner and hence the determination of TNT explosion overpressure is only a function of stand-off distance in the space.

For gas explosions, the pressure time history is typically a triangular shaped wave with an extremely short time period, (Fig. 7). For each monitor point, the impulse vs. time data obtained by means of integration of the pressure time history and this seen in Fig. 8. The maximum impulse is observed after the peak of the overpressure and the steady state of the impulse is seen after the pressure attenuates to $0 \mathrm{kPa}$.

By applying the data above to the structural members, the calculation of the final states of damage, which is of major concern can be assessed. Specifically, a structural member in an offshore module subjected to gas explosion is simplified as a Single Degree Of Freedom (SDOF) equivalent structural model to assess its structural response behaviour. The maximum deflection rather than the detailed deflection-time history of the structure determines the failure criterion of the structure.

In order to evaluate the structural damage level, a pressure-impulse ( $p$-i) diagram of the equivalent SDOF structural model (Mays \& Smith, 1995; Smith \& Hetherington, 1994) was developed as shown in Fig. 9. Once the critical deflection (maximum allowable deflection) $y_{c}$ of the structure is specified, a curve was obtained, as the dashed line shows in Fig. 9, which indicates various combinations of the non-dimensional initial peak overpressure $p$ and the impulse $i$ of the external load that will cause the same deflection of the structure. The non-dimensional pressure and impulse are defined as $p=P_{o} A /\left(k y_{c} / 2\right)$ and $i=I_{o} / y_{c} \sqrt{k m_{s e}}$. 
390 The impulsive asymptote of the curve is $i=1.0$ and the quasi-static asymptote is $p=1.0$.

$391 P_{o}$ is the initial peak pressure of the blast load and $I_{o}$ is the impulse of the blast load as 392 shown in Fig. 8, $A$ is the cross-sectional area of the SDOF structural, $m_{s e}$ is the 393 equivalent mass of the equivalent SDOF structure and $k$ is its stiffness. In this study, 394 we take the gas explosion scenarios at the four monitor points in the congested 395 configuration as examples, the steel material was used to simulate the offshore 396 structural members which are modelled as simply supported beams, the cross-sectional 397 area, the equivalent mass and the stiffness were set as $1 \mathrm{~m}^{2}, 1 \mathrm{~kg}$ and $3 \times 10^{6} \mathrm{~N} / \mathrm{m}$. 398 Therefore, the $p-i$ combinations of the gas explosion blast load were determined; the 399 four points indicated in Fig. 9 represent the blast load results obtained in Fig. 8. For the 400 four monitor points, any data below the dashed curve (overpressure and impulse at point 4011 and point 2) will not result in any damage of the structure while those above the curve 402 (overpressure and impulse at point 3 and point 4) will induce failure of the structure.

\section{Conclusion}

This paper examined 400 scenarios in geometries similar to the MERGE experiments on which the GAME correlation is based, with one important distinction: The confinement and congestion were deliberately varied such that some of the geometries 408 possessed inhomogeneity of both parameters. Little experimental data exists for such configurations and hence the cases were modelled here with the commercial CFD software FLACS (GexCon, 2011). A realistic model was also examined and modelled using the commercial code. Realistic geometries also typically display a high degree of

412 inhomogeneity in confinement and congestion. 
414 The overpressure predictions using FLACS (GexCon, 2011) at various target locations

415 were compared with the results from a newly derived correlation by (Li et al., 2014)

416 and the GAME correlation. It is found that the CSC correlation better agrees with the

417 overpressure predictions obtained using CFD when compared with the GAME

418 correlation. The results further demonstrate that the correlation by the CSC is suitable

419 for the modelling of realistic geometries.

420

421 The numerically calculated pressure and impulse vs. time results were related to

422 damage level by simplifying the offshore structural component as an SDOF equivalent

423 model, the structural damage level was determined within the $p$ - $i$ diagram. The results

424 show that the cases examined are ones that require an increased level of accuracy as

425 they are very close to cases that may cause permanent damage to structural members.

426 


\section{References}

Arntzen, B. J. (1998). Modelling of turbulence and combustion for simulation of gas explosions in complex geometries. Ph.D. Thesis, The Norwegian University Norway.

Bjerketvedt, D., Bakke, J. R., \& vanWingerden, K. (1997). Gas explosion handbook. Journal of Hazardous Materials, 52(1), 1-150.

Eggen, J. B. M. M. (1995). GAME: development of guidance for the application of the Multi-Energy Method. Rijswijk: HSE Books.

EMEG. (1997). Explosion Model Evaluation Group, Specifications of test cases for gas explosions - test case C1. EME project, DGXII, Brussels, Belgium.

Ferrara, G., Di Benedetto, A., Salzano, E., \& Russo, G. (2006). CFD analysis of gas explosions vented through relief pipes. Journal of Hazardous Materials, 137(2), 654-665. doi: DOI 10.1016/j.jhazmat.2006.03.037

GexCon. (2011). FLACS v9.1 User's Manual. Norway: Doxygen.

Harris, R. J., \& Wickens, M. J. (1989). Understanding vapour cloud explosions - an experimental study. Paper presented at the 55th Autumn meeting, the Institution of Gas Engineers, Kensington, UK.

Hjertager, B. H. (1984). Computer-Simulation of Turbulent Reactive Gas-Dynamics. Modeling Identification and Control, 5(4), 211-236. 
Hjertager, B. H. (1993). Computer Modeling of Turbulent Gas-Explosions in Complex 2d and 3d Geometries. Journal of Hazardous Materials, 34(2), 173197. doi: Doi 10.1016/0304-3894(93)85004-X

Li, J., Abdel-jawad, M., \& Ma, G. (2014). New correlation for vapor cloud explosion overpressure calculation at Congested Configurations. Journal of Loss Prevention in the Process Industries, 31, 16-25. doi: 10.1016/j.jlp.2014.05.013

Marangon, A., Carcassi, M., Engebo, A., \& Nilsen, S. (2007). Safety distances: Definition and values. International Journal of Hydrogen Energy, 32(13), 2192-2197. doi: DOI 10.1016/j.ijhydene.2007.04.007

Mays, G. C., \& Smith, P. D. (1995). Blast effects on buildings - Design of buildings to optimize resistance to blast loading. London, UK: Thomas Telford Publications.

Mercx, W. P. M., Johnson, D. M., \& Puttock, J. (1995). Validation of Scaling Techniques for Experimental Vapor Cloud Explosion Investigations. Process Safety Progress, 14(2), 120-130. doi: DOI 10.1002/prs.680140206

Mercx, W. P. M., \& van den Berg, A. C. (2005). Chapter 5: Vapour Cloud Explosion, TNO Yellow Book: Methods for the calculation of physical effects due to releases of hazardous materials (2nd Edition). Rijswijk, The Netherlands.

Patankar, S. V. (1980). Numerical heat transfer and fluid flow. London: Hemisphere publishing corporation. 
Schumann, S., Haas, W., \& Schmittberger, H. (1993). Dust Explosion Venting Investigation of the Secondary Explosion for Vessel Volumes from $0.3 \mathrm{M}(3)$ to 250 M(3). Staub Reinhaltung Der Luft, 53(12), 445-451.

Smith, P. D., \& Hetherington, J. G. (1994). Blast and ballistic loading of structures. Oxford, UK: Butterworth-Heinemann.

Vandenberg, A. C. (1985). The Multi-Energy Method - a Framework for Vapor Cloud Explosion Blast Prediction. Journal of Hazardous Materials, 12(1), 1-10.

Wingerden, C. J. M. v. (1988). Investigation into the blast produced by vapour cloud explosions in partially confined areas. Rijswijk, The Netherlands: HSE Books

\section{Wingerden, C. J. M. v. (1989). Experimental investigation into the strength of blast} waves generated by vapour cloud explosions in congested areas. Paper presented at the 6th Int. Symp.'Loss Prevention and Safety Promotion in the Process Industries', Oslo, Norway. 


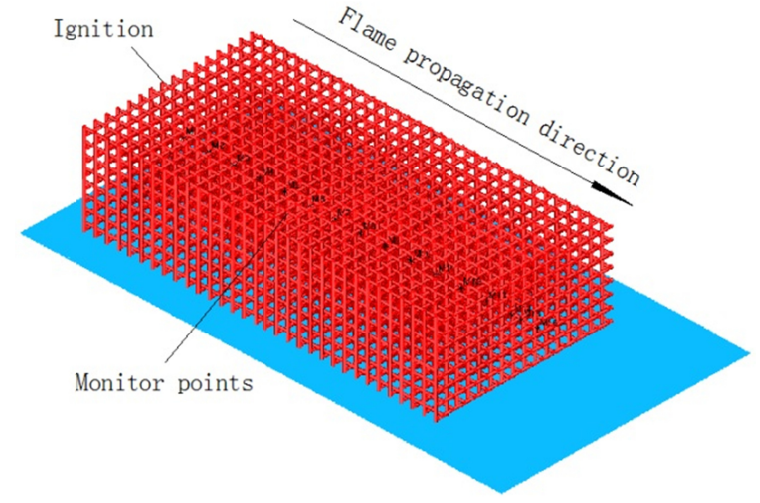

(a) Artificial Model

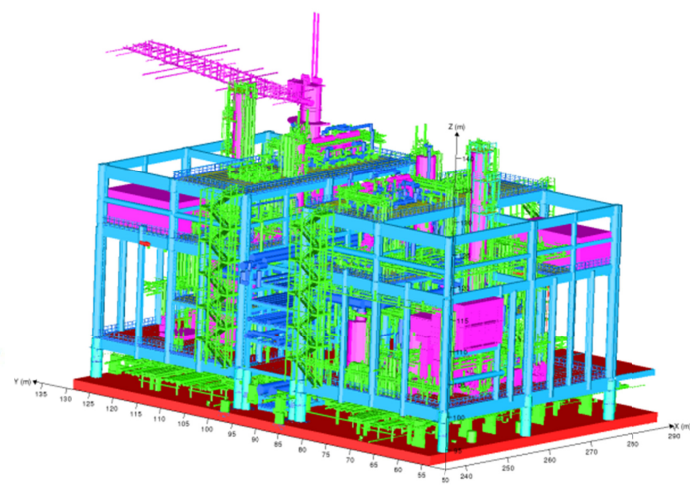

(b) Realistic Model

Fig. 1 FLACS simulation models 


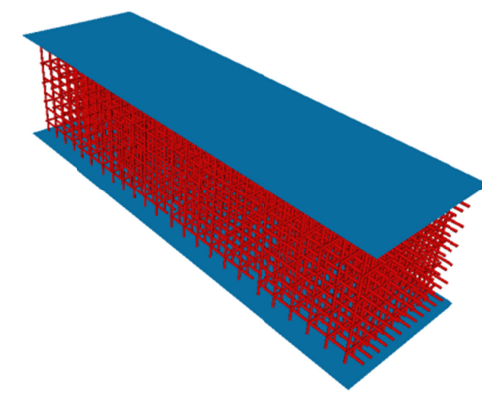

(a) Fully confined module

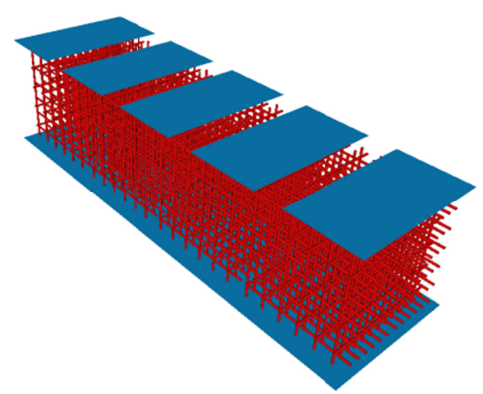

(b) partially confined module

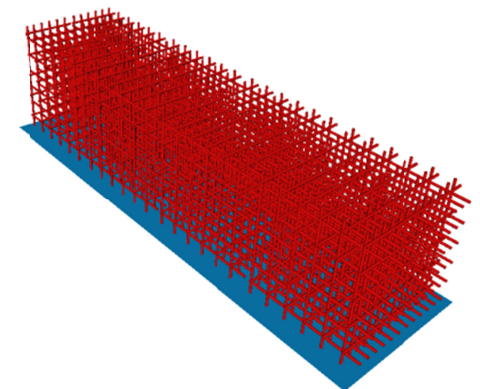

(c) Open in +z-direction

Fig. 2 Artificial modules with varying confinement 


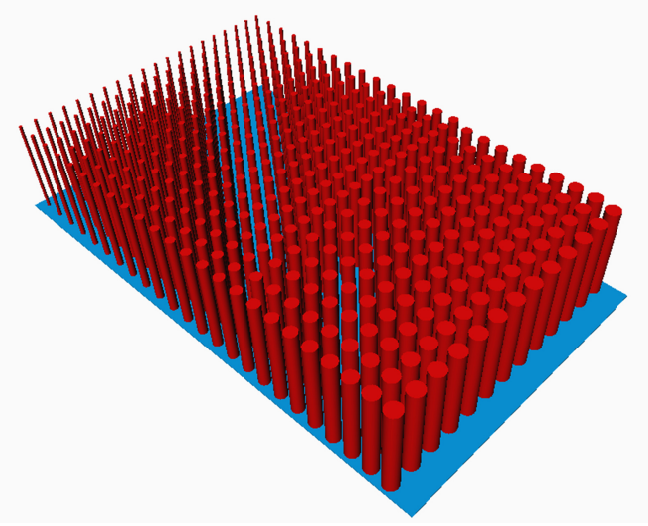

Module 1 - Irregular-arranged

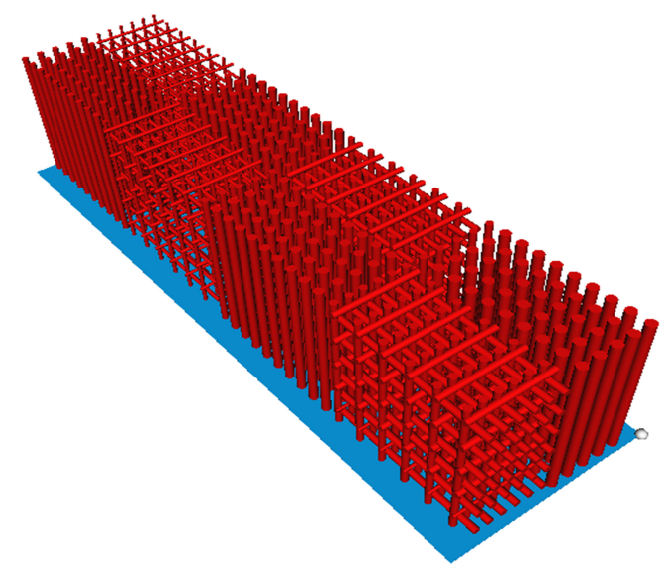

Module 3 - Irregular-arranged

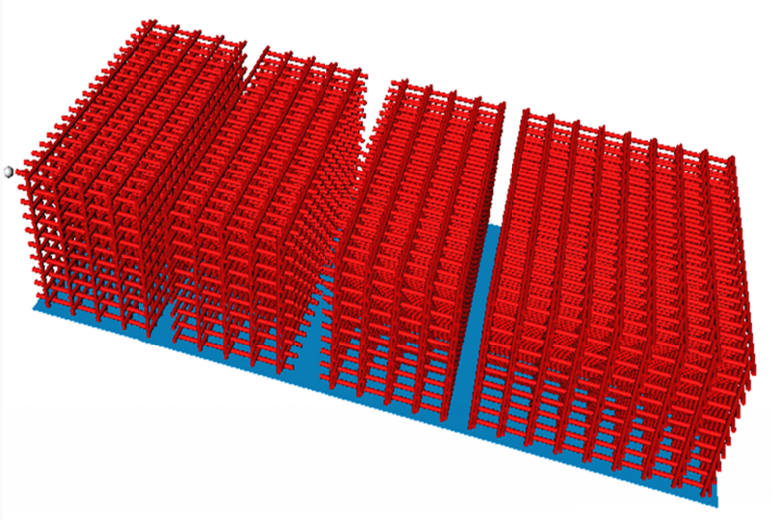

Module 2-Irregular-arranged

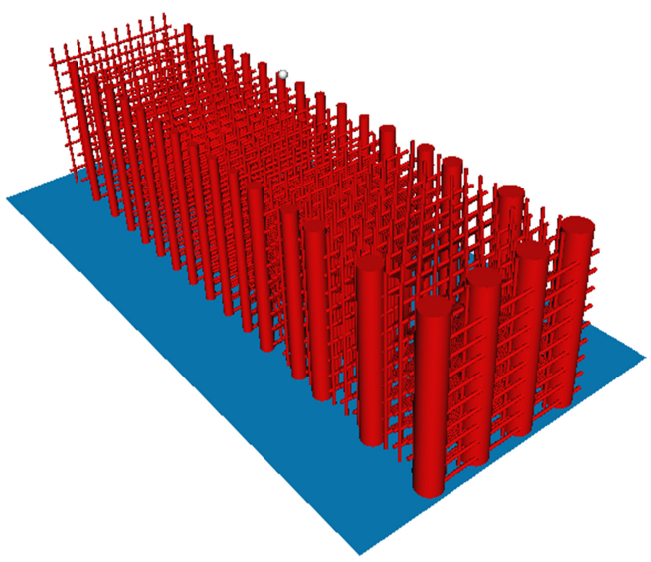

Module 4 - Irregular-arranged

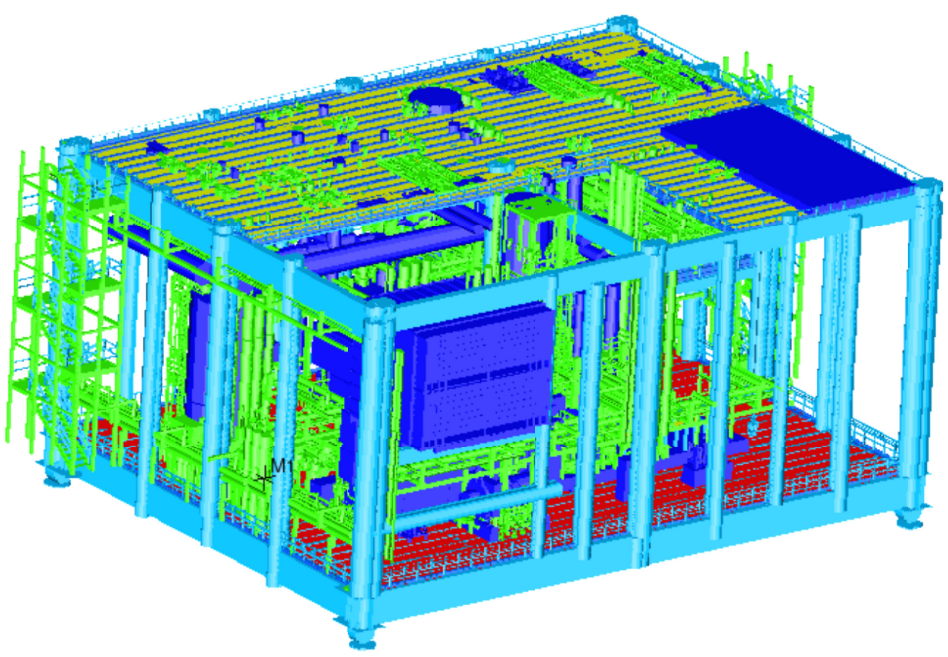

Module 5 - Realistic Module

Fig. 3 Modules with irregularities 1-5 

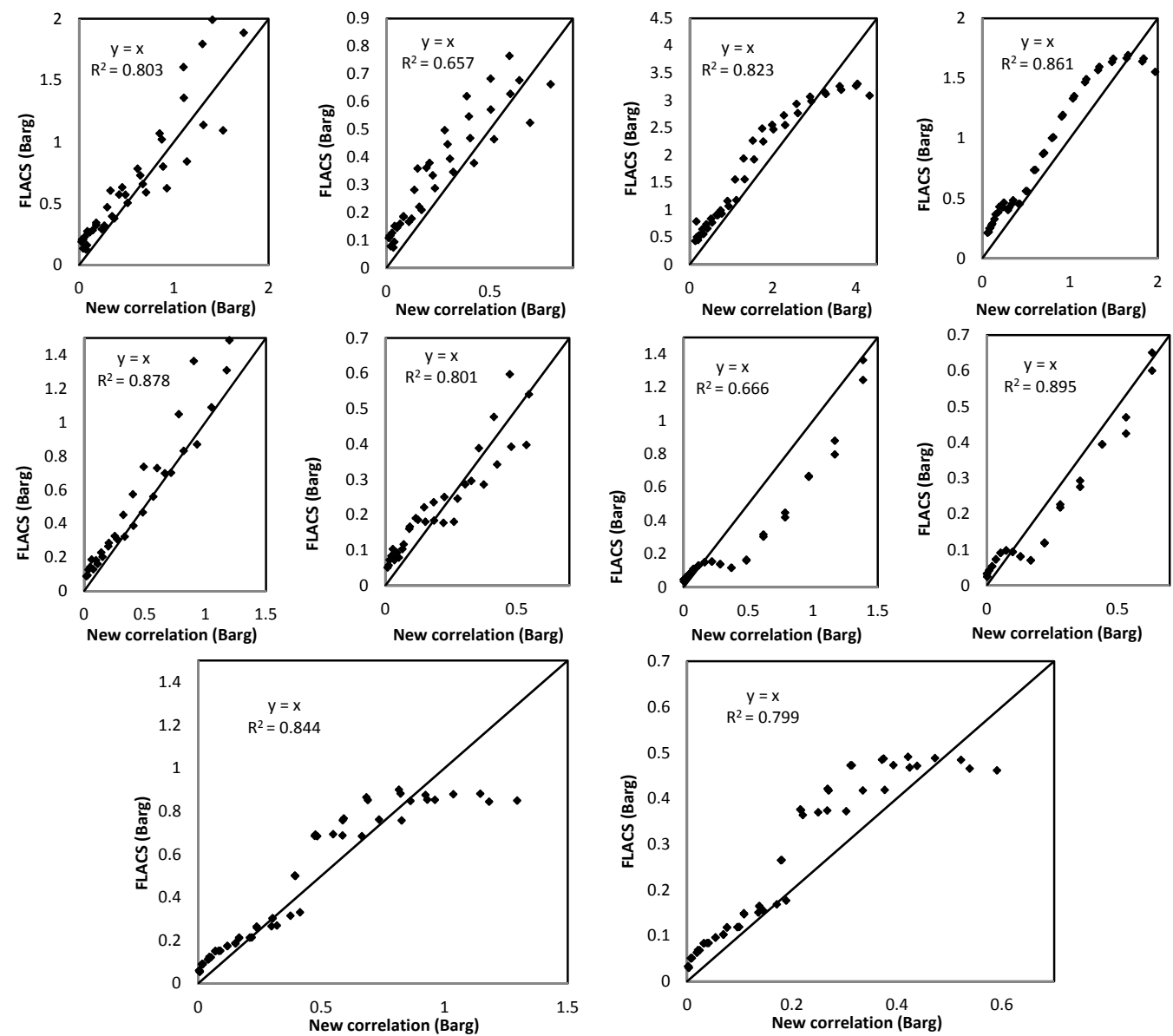

Fig. 4 the comparison of CSC correlation overpressure data vs. FLACS results for the irregular-patterned configurations subject to methane and propane vapour explosions 


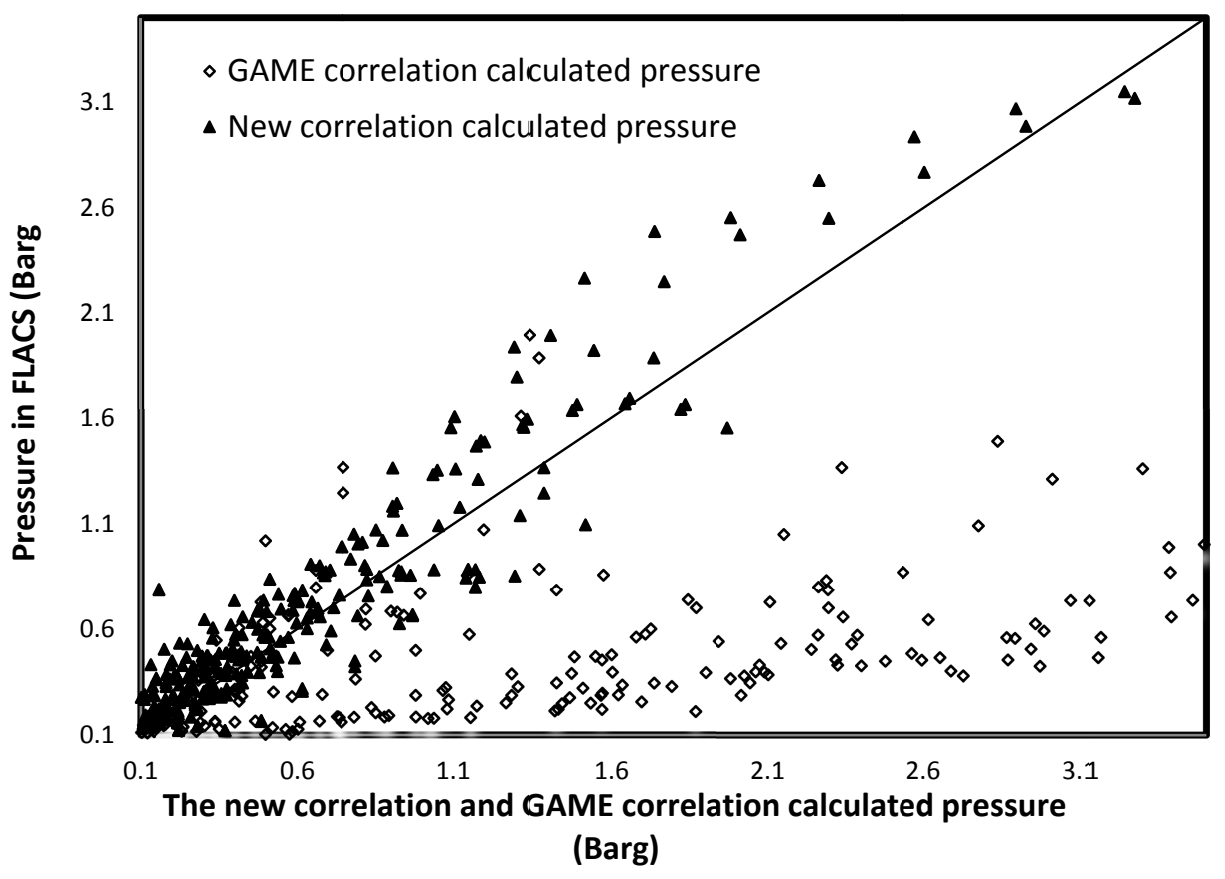

Fig. 5 the comparison of the new correlation and the GAME overpressure data vs. FLACS results for the irregular-patterned configurations subject to methane and propane vapour explosions 


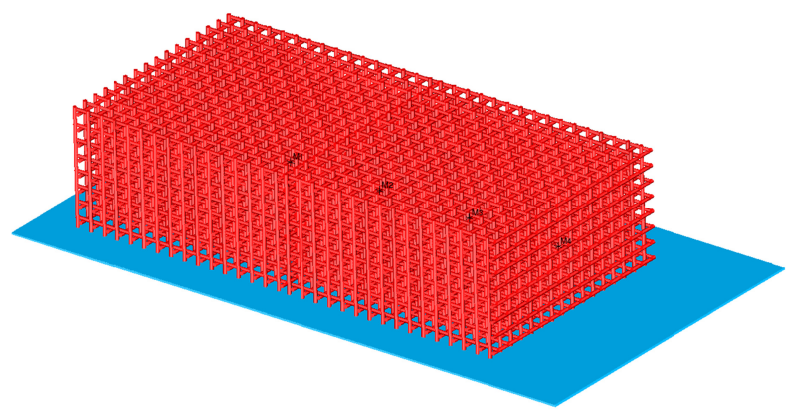

(a) Monitors within the congestion

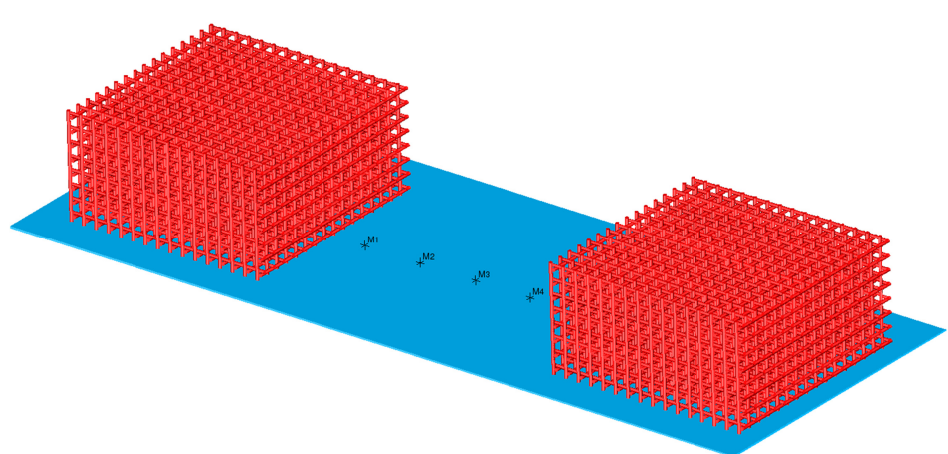

(b) Monitors in the open space

Fig. 6 Specified monitor points at different gas explosion scenarios 


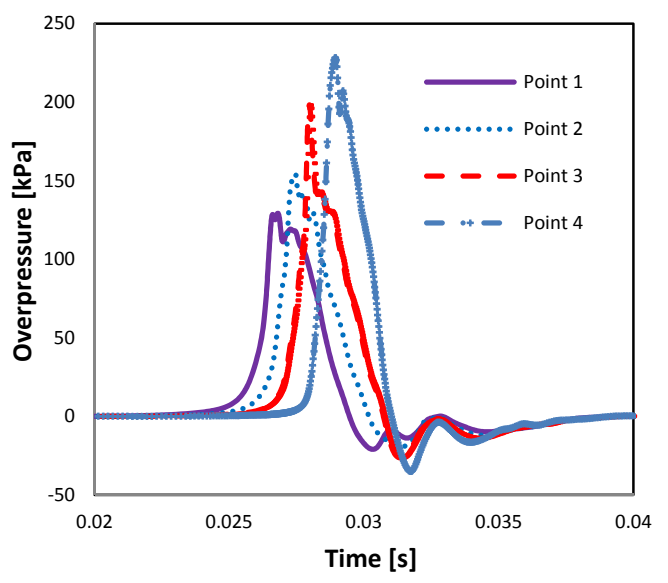

(a) Monitors within the congestion

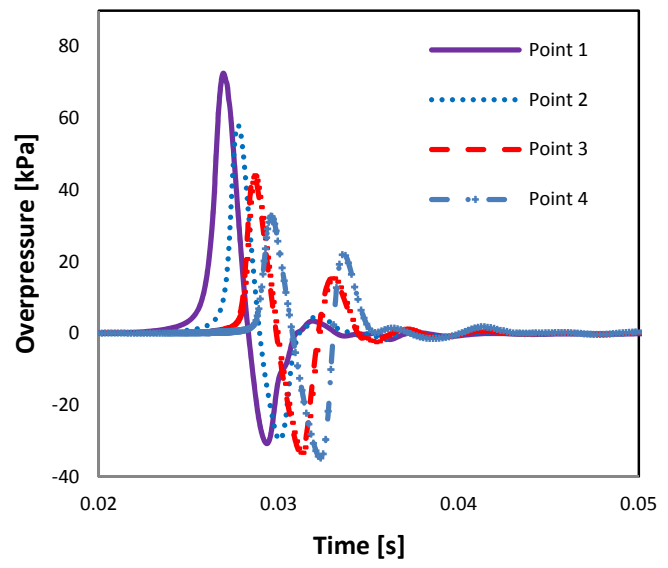

(b) Monitors in the open space

Fig. 7 The overpressure vs. time results for the specified monitor points 


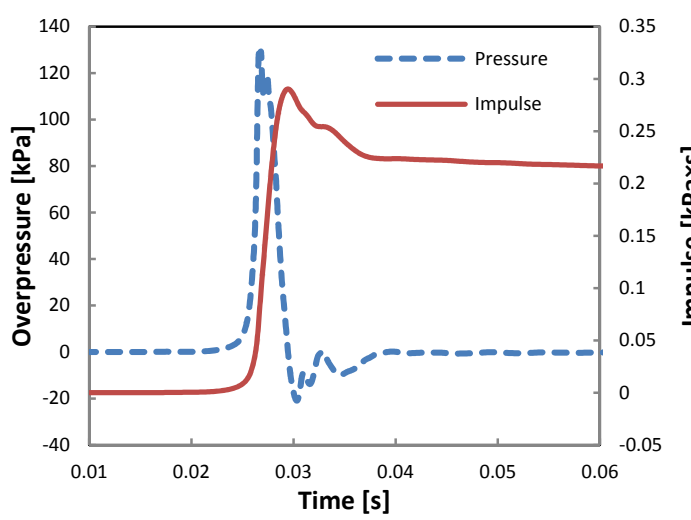

(a) Monitor point 1

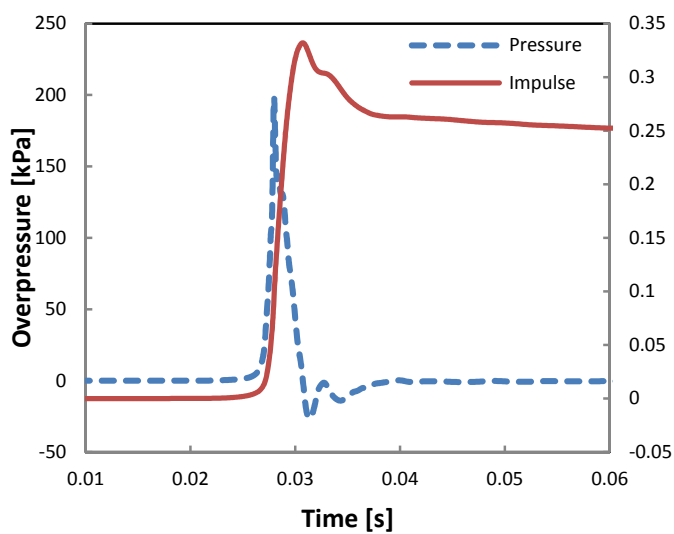

(c) Monitor point 3

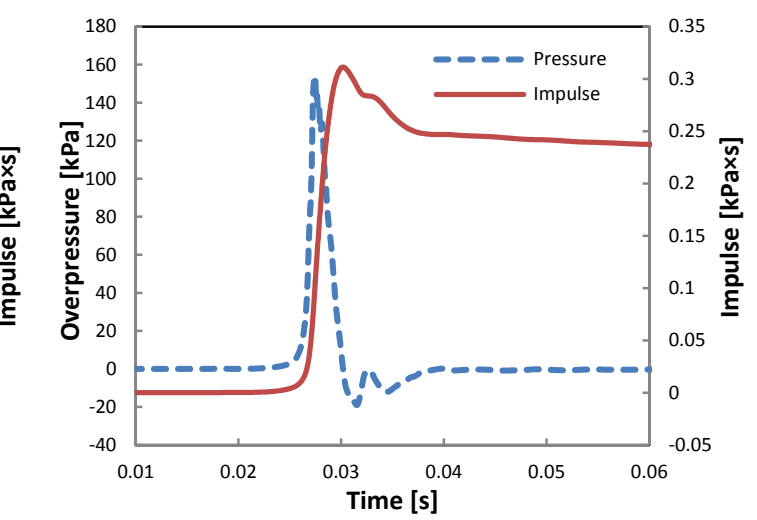

(b) Monitor point 2

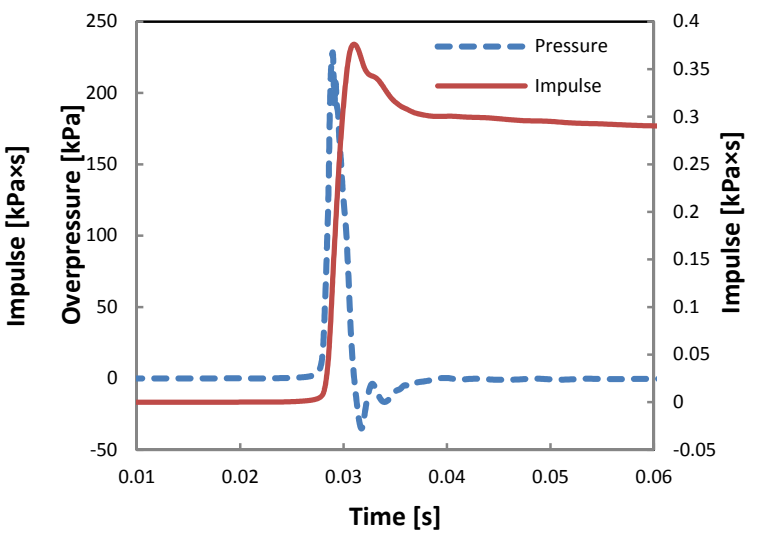

(d) Monitor point 4

Fig. 8 The time domain results of overpressure and impulse at different monitor points in the congested space 


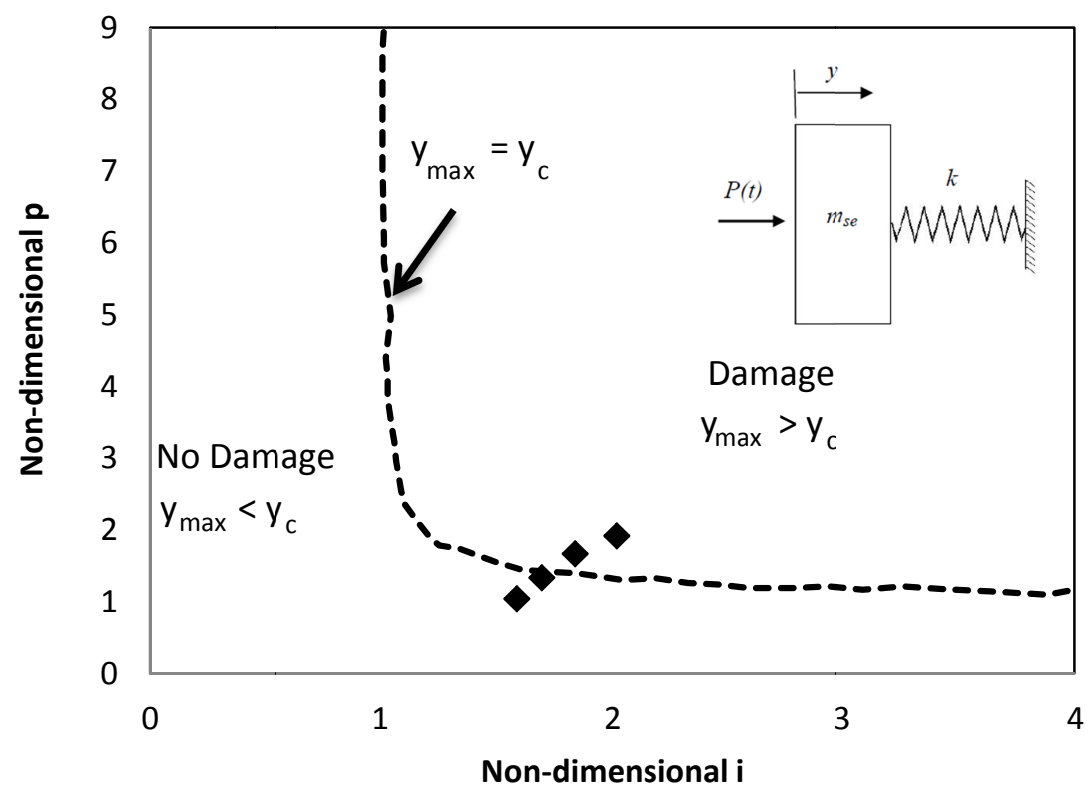

Fig. 9 Non-dimensional $p-i$ diagram of an equivalent SDOF structural model 


\section{Figure captions list}

Fig. 1 FLACS simulation models

Fig. 2 Artificial modules with varying confinement

Fig. 3 Modules with irregularities 1-5

Fig. 4 the comparison of CSC correlation overpressure data vs. FLACS results for the irregular-patterned configurations subject to methane and propane vapour explosions Fig. 5 the comparison of the new correlation and the GAME overpressure data vs. FLACS results for the irregular-patterned configurations subject to methane and propane vapour explosions

Fig. 6 Specified monitor points at different gas explosion scenarios

Fig. 7 The overpressure vs. time results for the specified monitor points

Fig. 8 The time domain results of overpressure and impulse at different monitor points in the congested space

Fig. 9 Non-dimensional p-i diagram of an equivalent SDOF structural model 
Table 1 Parameters in difference modules

\begin{tabular}{llllccc}
\hline Case No. & Gas composition & D $(\mathbf{m})$ & VBR $^{*}$ & $\mathbf{S}_{\mathbf{l}}(\mathbf{m} / \mathbf{s})$ & Gas density $\left(\mathbf{k g} / \mathbf{m}^{\mathbf{3}}\right)$ & $\mathbf{C}_{\mathbf{m}}$ \\
\hline 1. Module 1 & Pure Methane & 0.37 & 0.11 & 0.40 & 0.65 & 1.00 \\
2. Module 1 & Pure Propane & 0.37 & 0.11 & 0.46 & 1.80 & 1.00 \\
3. Module 2 & Pure Methane & 0.31 & 0.14 & 0.40 & 0.65 & 0.96 \\
4. Module 2 & Pure Propane & 0.31 & 0.14 & 0.46 & 1.80 & 0.96 \\
5. Module 3 & Pure Methane & 0.33 & 0.13 & 0.40 & 0.65 & 0.90 \\
6. Module 3 & Pure Propane & 0.33 & 0.13 & 0.46 & 0.65 & 0.90 \\
7. Module 4 & Pure Methane & 0.21 & 0.04 & 0.40 & 1.80 & 0.90 \\
8. Module 4 & Pure Propane & 0.21 & 0.04 & 0.46 & 0.65 & 0.76 \\
9. Module 5 & Pure Methane & 0.59 & 0.12 & 0.40 & 1.80 & 0.76 \\
10. Module 5 & Pure Propane & 0.59 & 0.12 & 0.46 &
\end{tabular}

$*$ VBR here is the volume blockage ratio of the entire obstructed region for Module 1 to 5. 